\title{
A Child with Influenza A and Large Pneumatocele
}

\author{
Seyedeh Masumeh Hashemi ${ }^{1}$, Azita Behzad ${ }^{1}$, Seyyedeh Narjes Ahmadizadeh ${ }^{1}$ and Fariba Shirvani ${ }^{2, *}$ \\ ${ }^{1}$ Pediatric Intensive Care Departmant, Mofid Children Hospital, Shahid Beheshti University of Medical Sciences, Tehran, Iran \\ ${ }^{2}$ Pediatric Infections Research Center, Research Institude for Children's Health, Shahid Beheshti University of Medical Sciences, Tehran, Iran \\ "Corresponding author: Pediatric Infections Research Center, Research Institute for Children Health, Shahid Beheshti University of Medical Sciences, Tehran, Iran. Email: \\ shirvanifariba@rocketmail.com
}

Received 2020 November 18; Revised 2020 December 29; Accepted 2021 January 16.

\begin{abstract}
Necrotizing pneumonia (NP) is a rare complication of community-acquired pneumonia, which occurs in patients with viral pneumonia such as influenza and secondary bacterial infection. We present a five-year-old boy with cough and dyspnea and low $\mathrm{SpO}_{2}$, who was admitted to PICU. He was intubated, and two-sided chest tubes were placed because of pleural effusion. Nasopharyngeal RT-PCR for H1N1 was positive. Subcutaneous and mediastinal emphysema and a large pneumatocele developed concomitantly, and the patient underwent three times percutaneous aspiration of pneumatocele under anesthesia and CT scan guide without surgery. The size of the pneumatocele decreased, and the patient was extubated. After one month of admission, he was discharged in good condition and no pulmonary sequela.
\end{abstract}

Keywords: Child, Influenza Virus, Pneumonia, Necrotizing

\section{Introduction}

Necrotizing pneumonia (NP) is a rare complication of community-acquired pneumonia, and despite the advance in antimicrobial therapy, the incidence is increasing. Necrotizing pneumonia was described in children in 1994 when a four-case case series was published (1). In most cases, the associated bacteria consist of Staphylococcus, Pneumococcus, Haemophilus influenza, Enterobacter cloacae, Escherichia coli, Klebsiella pneumoniae, and Pseudomonas aeruginosa $(2,3)$ or even fungi such as Histoplasma Capsulatum (4). Necrotizing pneumonia is placed in the spectrum between pulmonary gangrene and pulmonary abscess and frequently is accompanied by bronchopleural fistula and empyema (5). There are several ways to manage pneumothorax, emphysema, empyema, pneumatocele, and Bronchopulmonary fistula. Generally, it is recommended to minimize surgical intervention to avoid bronchopleural fistula (BPF) (6). In some situations, such as sustained air leak, loculated empyema with no response to chest tube, or pulmonary tissue necrosis, surgery is inevitable (5). We introduce a five-year-old boy with necrotizing pneumonia due to influenza with mediastinal and soft tissue emphysema, pneumothorax, and pneumatocele who was treated with intermittent chest tube suctioning and no invasive surgical intervention.

\section{Case Presentation}

A five-year-old boy was admitted to the emergency department with respiratory distress. There was a history of 10 days of cough, dyspnea, and fever. He was examined by a physician, and azithromycin and cefixime were prescribed with no improvement. He was hospitalized with a respiratory rate of $60 / \mathrm{min}$ and $\mathrm{O}_{2}$ saturation of $80 \%$ at room temperature. Two-sided pleural effusion was found in the lungs by radiography, and he was immediately referred to the operation room, and both sides chest tubes were inserted. Because of low blood pressure and poor condition, he was intubated and admitted to ICU with the diagnosis of sepsis and moderate ARDS $\left(\mathrm{PaO}_{2}=65\right.$ $\mathrm{mmHg}, \mathrm{FiO}_{2}=60 \%, \mathrm{PEEP}=5 \mathrm{~cm} \mathrm{H}_{2} \mathrm{O}$ ) (based on ARDS Berlin definition) (7) and two sides chest tubes were inserted, and antibiotic and IV fluid were used. Echocardiography showed reduced ejection fraction (40\%), and epinephrine was started. There was purulent and serosanguinose fluid drainage from both chest tubes. Nasopharyngeal suction and BAL PCR were positive for H1N1 influenza, while negative for influenza $\mathrm{B}$. Moreover, $\mathrm{CBC}$ showed $\mathrm{WBC}=$ $17800 / \mathrm{mm}^{3}$ (normal range $5000-10000 / \mathrm{mm}^{2}$ ), HG $=9.9$ $\mathrm{mg} / \mathrm{dL}(10.9-15 \mathrm{mg} / \mathrm{dL}), \mathrm{N}=89 \%, \mathrm{~L}=11 \%, \mathrm{ESR}=85, \mathrm{AST}=45$ $\mathrm{U} / \mathrm{dL}(8-48 \mathrm{U} / \mathrm{dL})$, and ALT $=39 \mathrm{U} / \mathrm{dL}(7-55 \mathrm{U} / \mathrm{dL})$. In pleural fluid Glu $=157 \mathrm{mg} / \mathrm{dL}(70-130 \mathrm{mg} / \mathrm{dL})$, Pro $=2400 \mathrm{mg} / \mathrm{dL}(1$ $-2 \mathrm{~g} / \mathrm{dL}), \mathrm{WBC}=15200 / \mathrm{mm}^{2}\left(<1000 / \mathrm{mm}^{2}=\right.$ transudate $), \mathrm{P}$ 
$=85 \%, \mathrm{~L}=15 \%, \mathrm{RBC}=780 / \mathrm{mm}^{2}, \mathrm{LDH}=1574 \mathrm{mg} / \mathrm{dL}$ ( $<50 \%$ of plasma $\mathrm{LDH}), \mathrm{ADA}=9 \mathrm{IU} / \mathrm{mL}$ ( $<36$ for tuberculosis), serum pro $=4.6 \mathrm{~g} / \mathrm{dL}(3.5-\mathrm{g} / \mathrm{dL})$. During the treatment process, the patient developed subcutaneous and mediastinal emphysema. Also, CT scan showed a large air containing cystic lesion (pneumatocele) in posterior aspect of left hemithorax, large bulla formation or loculated pneumothorax in the hemithorax with possible collapse of underlying lung in posterior segment of left lower lobe, and peribronchial infiltration was noted in RLL and LLL (Figure 1).

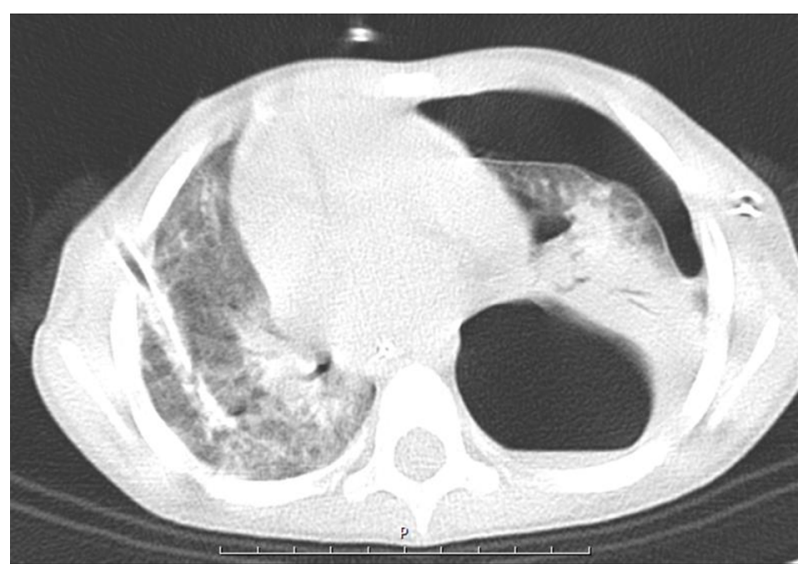

Figure 1. Large Left Side Pneumatocele with Air Fluid Level and Pneumothorax

Three times percutaneous aspiration of pneumatocele was done under anesthesia and CT scan guide without surgery (Figure 2). Owing to the fact that pneumatocele was large and had a compressive effect and did not respond with two times percutaneous aspiration, the need for surgery was felt; however, the third time aspiration trial was attempted, and the size of pneumatocele decreased gradually, and the patient was extubated. The patient received three times IVIG because of low level of immunoglobulins, IgE $=46 \mathrm{kU} / \mathrm{L}(0-148) \mathrm{kU} / \mathrm{L}, \operatorname{IgG}=$ $438 \mathrm{mg} / \mathrm{dL}(600-1660 \mathrm{mg} / \mathrm{dL}), \mathrm{IgA}=59 \mathrm{mg} / \mathrm{dL}(70-400$ $\mathrm{mg} / \mathrm{dL}), \operatorname{IgM}=42 \mathrm{mg} / \mathrm{dL}(96.6 \pm 50 \mathrm{mg} / \mathrm{dL}), \operatorname{IgE}=46(20$ $=100 \mathrm{IU} / \mathrm{mL})$. Diphtheria antibody $=0.31 \mathrm{IU} / \mathrm{mL}(<0.1$ basic immunization), anti-tetanus $\mathrm{Ab}=2.8 \mathrm{IU} / \mathrm{mL}$. Further, CD4 blood counts $=33 \%(22-62 \%)$, CD8 $=14 \%(12-36 \%)$, CD56 = $1.42(4-105)$, CD4/CD8 = 2.35 (1 - 3), and nitroblue tetrazolium $(\mathrm{NBT})=100 \%(90 \%-100 \%)$. The tracheal and pleural fluid culture was Acinetobacter-positive that was resistant to amikacin, cefepime, ciprofloxacin, and meropenem. Blood culture was positive for Pseudomonas sensitive to amikacin, ciprofloxacin, gentamycin and resistant to cefepime and imipenem. The patient received tazocin, ciprofloxacin, linezolid, colistin, and vancomycin in the course of his treatment. This patient suffered from the complication of necrotizing pneumonia and pneumothorax with complication of septic pleural effusion. Ventilator setup was based on open lung strategy and permissive hypercarbia and hypoxia. He was intubated for 10 days, and 48 hours of cisatracurium drip and 10 $\mathrm{mg} / \mathrm{kg} / \mathrm{h}$ magnesium sulfate and corticosteroids were administered. The big pneumatocele was aspirated under the CT scan guide repeatedly without surgery, and air suctioning resulted in gradual improvement of his condition. Finally, after 15 days, a significant improvement was observed (Figure 3). He was discharged from the hospital in good condition, and there was no problem with follow-up visits.

\section{Discussion}

We presented a previously healthy child with H1N1positive necrotizing pneumonia. Viral pneumonia is rarely accompanied by necrotizing pneumonia, but the invasive nature of the influenza virus and secondary bacterial infection can cause this complication. There are reports on H1N1 necrotizing pneumonia in the literature (8-10). Our patient developed moderate ARDS $\left(\mathrm{PaO}_{2} / \mathrm{FiO}_{2}=108\right)$ characterized by lung edema and increased capillary permeability, and diffuse alveolar damage. The child recovered after 15 days of supportive therapy and intubation without the need for ECMO. In the 2009 H1N1 pandemic, 49 adult patients with confirmed H1N1-associated ARDS from 14 ICU centers in Italy received Veno-venous ECMO with a 71\% survival rate (11).

The tracheal and pleural fluid culture was Acinetobacter, and it seems the patient developed secondary nosocomial pneumonia, but there is a possibility of false-positive results due to contamination. In a three-year-old girl with ARDS and H1N1 necrotizing pneumonia (2009) (Korea), ECMO rescued the patient, and tracheal and pleural effusion culture was Stenotrophomonas maltophilia and Acinetobacter baumannii due to nosocomial infection (12).

Our patient developed subcutaneous and mediastinal emphysema, possibly due to increased lung pressures, which causes alveoli to rupture. Spontaneous mediastinal and subcutaneous emphysema are reported in three children with H1N1 pneumonia during the 2009 pandemic (Ottawa) (8). Our patient was studied for possible immunodeficiency, and lower than normal immunoglobulin level was detected. In a study of 80 children with communityacquired pneumonia from Boston (1990 - 2005), two cases with underlying immunodeficiency were detected, one with Schwachman-Diamond syndrome and the other with chronic granulomatous disease (CGD) (13). Underlying immunodeficiency is not common in patients with necrotizing pneumonia except for patients with a history of 


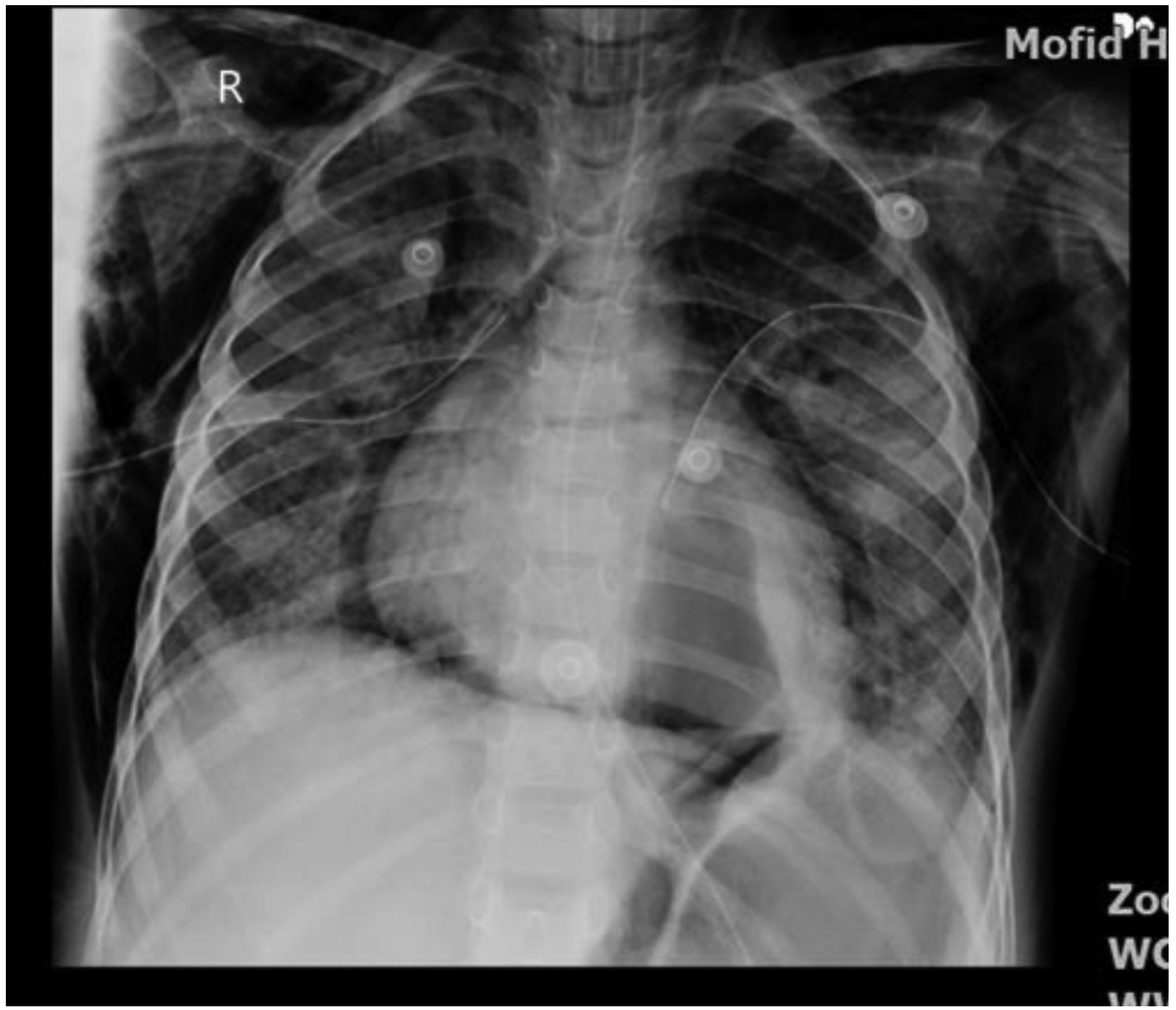

Figure 2. Mediastinal emphysema and left side pneumothorax and pneumatocele

recurrent, persistent, or severe infection, especially with catalase-positive or opportunistic and unusual organisms (5). Our patient had no history of previous admission or important infectious disease. Laboratory reexamination was normal after discharge, and he is in good condition.

Surgical intervention is a popular approach to treat pneumatocele. In a study on 26 pediatric cases with necrotizing pneumonia (2006 - 2016, Brazil), debridement and decortication of necrotic areas were performed in 23 cases and lobectomy in three cases, preoperative chest drainage was performed in 24 cases, but postoperative bronchopulmonary fistula occurred as a complication in eight cases (14). The decision for surgery in patients depends on their condition and response to chest tube insertion. A
4 years old boy with S. pneumonia necrotizing pneumonia underwent left chest tube insertion, but he developed huge pneumatocele and Veno-venous ECMO, and rightsided lobectomy was performed for him (2019, Canada) (15).

A previously healthy nine-year-old girl was admitted to a hospital (2018, Bosnia and Herzegovina) with left pulmonary pleural effusion and necrosis and pancreatic pseudocyst who was treated with chest tube and ERCP and percutaneous drainage of pancreatic pseudocyst (2). Chest tube drainage was performed on loculated rightsided pneumothorax with persistent cystic lesions and H1N1 necrotizing pneumonia complicated with Staphylococcus aureus (2010) in a 32-year-old man (2010, Mexico) (8). 


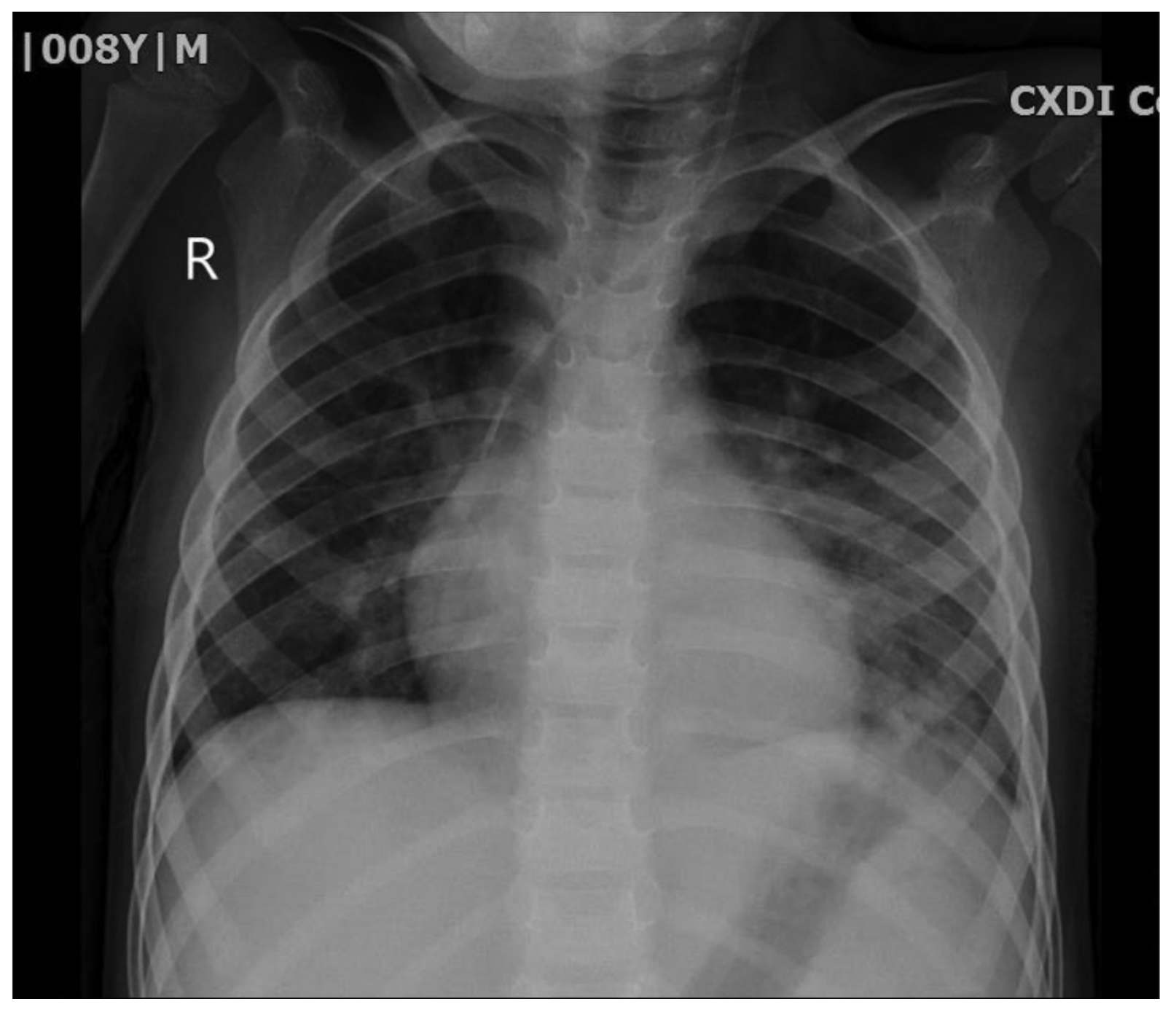

Figure 3. The near normal chest X-ray of patient after the therapy

The same plan was done in a five-year-old girl with H1N1 pneumonia complicated with S. pneumonia with rightsided pneumatocele and necrotic pleural effusion (2009, Canada) (9). Nonsurgical drainage can perform even in neonates, percutaneous pigtail catheter drainage was performed in a 13 days old 28 weeks neonate without fibrin sealant, chest tube and surgical resection (2019, USA) (16). There is no established algorithm to treat pneumatocele in cases of necrotizing pneumonia, but percutaneous CT-guided drainage under radiological control is recommended, and surgical intervention is suggested if this technique fails (3). Our patient experienced two times percutaneous drainage, and surgical intervention was indicated in his treatment process; however, the third trial of aspiration was attempted, and fortunately, it was suc- cessful. Surgical treatment is recommended to keep to a minimum, avoiding risk of bronchopleural fistula, but in some situations, such as tension pneumatocele, loculated empyema, especially if they lead to mass effect or causing hemodynamic instability, it is not possible (5). In this condition, surgery can be life-saving, and thoracotomy and drainage of supportive material and debridement should be done (17).

\subsection{Conclusions}

This case was treated with intermittent percutaneous drainage of pneumatocele under a CT scan guide with good results. It is recommended to withhold surgical treatment and thoracotomy if non-invasive procedures can be implemented. 


\section{Footnotes}

Authors' Contribution: $\mathrm{SH}, \mathrm{AB}$, and SA discussed the case and wrote the first draft and FSH edited the article.

Conflict of Interests: There was no conflict of interest.

Ethical Approval: Ethical approval was obtained from the Ethics Committee of Shahid Beheshti University of Medical Sciences and Health Services.

Funding/Support: There was no funding or support.

Informed Consent: Written informed consent was obtained from the patient's parents regarding the publication of this article.

\section{References}

1. Kerem E, Bar Ziv Y, Rudenski B, Katz S, Kleid D, Branski D. Bacteremic necrotizing pneumococcal pneumonia in children. Am J Respir Crit Care Med. 1994;149(1):242-4. doi: 10.1164/ajrccm.149.1.8111589. [PubMed: 8111589].

2. Selimovic A, Mujic E, Milišic S, Hasanbegovic E, Hasanbegovic S, Mušanovic S, et al. Pediatric necrotizing pneumonia: Case report and overview of conservative treatment. Int J Surg Med. 2019;5(3):169-71.

3. Tano KDOR, Stape A, Bousso A, Troster EJ, Milanez JR. Treatment of complicated pneumatocele. Einstein. 2005;3(2):115-8.

4. Schiavo DN, Bauer PR, Iyer VN, Ryu JH. Fatal fulminant necrotizing pneumonia: A case report. J Med Case Rep. 2014;8:37. doi: 10.1186/17521947-8-37. [PubMed: 24499506]. [PubMed Central: PMC3923567].

5. Masters IB, Isles AF, Grimwood K. Necrotizing pneumonia: An emerging problem in children? Pneumonia. 2017;25(9):11. doi:10.1186/s41479017-0035-0. [PubMed: 28770121]. [PubMed Central: PMC5525269].

6. Macedo M, Meyer KF, Oliveira TC. Necrotizing pneumonia in children submitted to thoracoscopy due to pleural empyema: Incidence, treatment and clinical evolution. J Bras Pneumol. 2010;36(3):301-5. doi: 10.1590/s1806-37132010000300006. [PubMed: 20625666].

7. Fanelli V, Vlachou A, Ghannadian S, Simonetti U, Slutsky AS, Zhang H. Acute respiratory distress syndrome: new definition, current and future therapeutic options. J Thorac Dis. 2013;5(3):326-34. doi: 10.3978/j.issn.2072-1439.2013.04.05. [PubMed: 23825769]. [PubMed Central: PMC3698298].
8. Udupa S, Hameed T, Kovesi T. Pneumomediastinum and subcutaneous emphysema associated with pandemic (H1N1) influenza in three children. CMAJ. 2011;183(2):220-2. doi: 10.1503/cmaj.100099. [PubMed: 21135087]. [PubMed Central: PMC3033927].

9. Morales-Blanhir J, Argote-Greene LM, Martin-Del-Campo LA, CarranzaMartinez I, Santillan-Doherty P. Parenchymal injury, fibrosis, and pneumatocele in an H1N1-positive patient. Chest. 2010;138(4):38A. doi: 10.1378/chest.10013.

10. Yazer J, Giacomantonio M, Macdonald N, Lopushinsky S. Severe necrotizing pneumonia in a child with pandemic (H1N1) influenza. CMAJ. 2011;183(2):215-9. doi: 10.1503/cmaj.100285. [PubMed: 21135076]. [PubMed Central: PMC3033926].

11. Pappalardo F, Pieri M, Greco T, Patroniti N, Pesenti A, Arcadipane A, et al. Predicting mortality risk in patients undergoing venovenous ECMO for ARDS due to influenza A (H1N1) pneumonia: The ECMOnet score. Intensive Care Med. 2013;39(2):275-81. doi: 10.1007/s00134-0122747-1. [PubMed: 23160769]. [PubMed Central: PMC7095375].

12. Ji S, Lee OJ, Yang JH, Ahn K, Cho J, Jeong SI, et al. 2009 H1N1 influenza virus infection and necrotizing pneumonia treated with extracorporeal membrane oxygenation. Korean JPediatr. 2011;54(8):3459. doi: 10.3345/kjp.2011.54.8.345. [PubMed: 22087202]. [PubMed Central: PMC3212705].

13. Sawicki GS, Lu FL, Valim C, Cleveland RH, Colin AA. Necrotising pneumonia is an increasingly detected complication of pneumonia in children. Eur Respir J. 2008;31(6):1285-91. doi: 10.1183/09031936.00099807. [PubMed: 18216055].

14. Dalponte RS, Heluany GCV, Michels M, Madeira K, Prado CE. Surgical treatment of necrotizing pneumonia in children: A 10-year assessment. Rev Col Bras Cir. 2020;47. e20202374. doi: 10.1590/0100-6991e20202374. [PubMed: 32236292].

15. Gerdung CA, Ross BC, Dicken BJ, Bjornson CL. Pneumonectomy in a child with multilobar pneumatocele secondary to necrotizing pneumonia: Case report and review of the literature. Case Rep Pediatr. 2019;2019:2464390. doi: 10.1155/2019/2464390. [PubMed: 31396429]. [PubMed Central: PMC6664550].

16. Munoz A, Moores DC, Khan FA, Baerg J, Radulescu A. Successful treatment of post-infectious pneumatocele via percutaneous drainage in a premature infant. J Pediatr Surg Case Rep. 2019;47:101235. doi: 10.1016/j.epsc.2019.101235.

17. DiBardino DJ, Espada R, Seu P, Goss JA. Management of complicated pneumatocele. J Thorac Cardiovasc Surg. 2003;126(3):859-61. doi: 10.1016/s0022-5223(03)00367-2. [PubMed: 14502169]. 